\title{
Parenting discipline styles and child psychopathology in a clinical sample of Egyptian children in the Nile Delta region
}

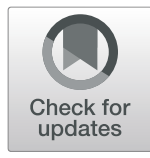

\author{
Mohammad A. Seleem * ${ }^{*}$ and Reham A. Amer
}

\begin{abstract}
Background: Few, if any, studies evaluated the effect of violent parenting discipline on children's psychological well-being in the Nile Delta region. The current study aimed to explore the spectrum of violent versus non-violent parenting discipline styles practiced by a sample of Egyptian families who came for psychiatric advice in Tanta Psychiatry and Neurology Center and the effects of such styles on youth's problem behavior and psychiatric diagnoses. Two hundred and seven families were recruited and interviewed using the Kiddie Schedule of affective disorders and schizophrenia-present and life-time version "K-SADS-PL." Arabic translated and validated versions of the conflict tactics scale-parent child version (CTS-PC) and the child behavioral checklist questionnaire (CBCL) for ages 6-18 years were used to evaluate parenting discipline styles and child psychopathology, respectively.

Results: Youth $(n=207)$ had an average age of $10.8 \pm 2.8$ years and $30 \%$ were females. A significant association was noted between all forms of psychological and physical violence against children, in addition to neglectful parenting, and both internalizing and externalizing problems in offspring with no significant differences between mild and severe forms of violence. Parents who were sexually abused as children had more tendency to practice violent discipline strategies on their own children.
\end{abstract}

Conclusions: All forms of child maltreatment, even mild forms, have a considerable effect on the child psychological development. National policies and legalization should be implemented to decrease child maltreatment and protect children from all forms of physical and sexual abuse.

Keywords: Parenting, Violent, Discipline, Child, Psychopathology, Nile, Delta

\section{Background}

Through the entire history of humankind, parenting discipline styles have been always playing a crucial contributing role in shaping the children's emotional wellbeing, cognitive capabilities, and behavioral patterns $[1,2]$. Numerous studies have documented the significant impact of parenting behavior in both normal and pathological behavior in children and adolescents [3-6]. There is consensus that the goal of parenting is to establish positive affective relationships with offspring with the ability to set appropriate limits that ensure the development of

\footnotetext{
* Correspondence: seleemm@med.tanta.edu.eg

Department of Psychiatry and Neurology, Faculty of Medicine, Tanta University, Tanta 31527, Egypt
}

\section{Springer Open}

affectively balanced and responsible personalities [7]. The authoritative style, which is considered the most balanced style among the three parenting styles described by Diana Baumrind [8], has the strongest evidence to have a very small to small negative associations with externalizing problems [7].

The precipitating effect of violent discipline styles on both internalizing and externalizing problems in offspring has been widely recognized $[1,4,6,7,9]$. Behaviors such as shouting, screaming, slapping, and beating the child are known to have a harmful effect on the child's behavior, self-esteem, and academic life [9]. However, such parenting behaviors are still widely practiced in many parts of the world, especially low- and middle- 
income countries. A recent large survey that screened 215,885 children in 62 countries showed that $43 \%$ of children were spanked, in the past month [10]. A considerable debate, among both researchers and the public, still exists about the differential effect of physical abuse and milder forms of corporal punishment on the child's well-being and behavioral outcomes. The effect sizes of both practices on detrimental child outcome did not differ significantly in a recent large meta-analysis [11]

The public view of violent discipline practices also varies across different cultures $[4,10,12]$. For instance, some studies report that physical discipline might be related to higher levels of externalizing behaviors for European American adolescents but lower levels of externalizing behaviors for African American youth [13]. In addition, Chao [14] reported that authoritative parenting styles have less favorable impact on school performance of Asian American youth than their European American peers.

In Middle Eastern Arab societies, many parents believe that harsh and restrictive parenting practices will have a positive impact on their children's behavior and their school achievement [15]. A recent survey done by the UNICEF in three big Egyptian cities revealed that most children surveyed are still exposed to physical violence, with more violence happening at home than in the street [16]. In a study conducted among Saudi female college students, the majority of the sample reported that they were physically punished at various stages in their life and justified the discipline [17]. Some reports suggested a significant effect of parental education, economic level, and urbanization on parenting styles and practices. More educated parents were reported to be less authoritarian and controlling than uneducated parents in Egypt [18], Saudi Arabia [19], and Algeria [20]. Few studies [21-23] explored the effect of violent discipline on Arab children and reported that authoritarian and violent parenting styles had deleterious effects on the child's psychological well-being and social adjustment. However, these studies mostly focused on small samples of children with no psychiatric diagnoses and did not use standardized psychometric tools in order to compare results to those in other cultures.

A previous report by our team [24] described a significant effect of both corporal punishment and physical abuse, reported by parents, on predicting depression, ADHD, and disruptive behavior disorders in children referred to our tertiary clinic. To our knowledge, this is the first study that aims to explore the full spectrum of violent versus non-violent styles of discipline practiced by parents and the potential effect of such styles on mental and psychological well-being of children and adolescents in a sample of youth referred for psychiatric evaluation in the Nile Delta region. We hypothesize that parenting styles that incorporate both psychological and physical aggression, widely accepted and practiced in our culture, will have a significant predictive effect on various internalizing and externalizing behavioral problems, in addition to specific psychiatric disorders, in this sample of Egyptian youth.

\section{Methods}

This cross-sectional study describes the parenting styles reported by the parents of 207 children and adolescents and their possible associations with the internalizing and externalizing problems and psychiatric diagnoses of this sample of Egyptian youth. The studied families were recruited from the Child and Adolescent Psychiatry Outpatient Clinic in Tanta Psychiatry and Neurology Center, one of the few tertiary centers for child and adolescent psychiatry in the Nile Delta, during a period from November 2017 to November 2018 after obtaining a written consent from parents and a verbal assent from the child prior to participation in the study. All children who attended to the clinic during that period and met the inclusion criteria for the study were included. The study was approved by the Ethical Research Committee of the Faculty of Medicine, Tanta University, under the code 31892/11/17.

To be recruited in the study, children's age had to range from 8 to 18 years, with a complaint of emotional and behavioral problems and with intelligence quotient (IQ) more than 70. Each recruited family had to have at least one parent with 12 years or more of education to be able to fill out the required questionnaires, which was a prerequisite for the inclusion in the study. Patients and/or parents who did not meet the inclusion criteria were excluded from the study. Exclusion criteria also involved children/adolescents who were previously diagnosed with intellectual disability, autism spectrum disorder, or psychotic disorders as children with these disorders might be especially difficult to manage and parents dealing with them might need specific psychometric tools to evaluate their parenting behavior. Parents who gave a history of being diagnosed with any psychiatric disorder (e.g., depression, bipolar, or substance abuse) were also excluded as their parenting practices might differ from healthy parents.

Demographic and socio-economic data were collected for each family by qualified social workers. A detailed developmental, medical, and family history were then obtained by a child psychiatrist including birth complications, developmental milestones, and family history of psychiatric illness. The Arabic version of the StanfordBinet Intelligence test - fourth edition [25, 26] was used to evaluate intellectual functions for all children. Socioeconomic statuses (SES) of the families have been evaluated using a standardized scale, namely the modified 
Fahmy and El-Sherbini scale for health research in Egypt $[27,28]$. The second stage was to verify the presence or absence of a psychiatric disorder among the children and adolescents by using the Kiddie Schedule of affective disorders and schizophrenia-present and life-time version "K-SADS-PL" [29], a semi-structured interview that takes $45-75$ min to administer. The Arabic translation of the K-SADS-PL used in the study has been validated on an Egyptian sample [30]. All the interviews were observed by competent interviewers and the diagnoses were confirmed by a qualified child and adolescent psychiatrist. Parenting discipline styles were evaluated using the conflict tactics scale-parent child version (CTS-PC) [31-33] which was previously translated and validated in Arabic by our team [34]. Both previous translation and current use of the scale were done after taking permission from the publisher of the scale ( $\mathrm{Li}$ cense WPS\#001530). The (CTS-PC) is a practical, quick, and widely accepted assessment tool for domestic violence directed toward children at home. It gives only subscores which include non-violent discipline, psychological aggression, and physical aggression which breaks into mild (corporal punishment), moderate (physical assault), and severe (physical maltreatment). It also gives scores for weekly discipline, neglect, and sexual abuse directed from anyone toward the child or the parent himself/herself. The main scores reflect parenting behaviors practiced during the last year, but lifetime scores are also given to reflect the possible use of the discipline style before the last year. No total score is calculated from the CTS-PC. The behavioral and emotional problems were explored using the Arabic version of the Child Behavioral Checklist Questionnaire (CBCL) for ages 6-18 years [35]. The Child Behavioral Checklist (CBCL) reflects the behavior of the child in the last 6 months and gives scores for competence, syndrome, and DSMoriented scales.

The sample size and power analysis were calculated using Epi-Info software statistical package created by the World Health Organization and Center for Disease Control and Prevention, Atlanta, Georgia, USA, version 2002. The sample size was calculated for a crosssectional study design and 95\% confidence interval. The expected association of parenting discipline styles and child psychopathology was $65 \%$ with a margin of error of 7 based on previous studies [1]. The sample size based on the previously mentioned criteria was found at $N>$ 178. It was increased by $15 \%$ to be 207 to compensate for missed information and improving the quality of data of the study. Data were fed to the computer and analyzed using IBM SPSS software package version 20.0 [36]. Simple descriptive analysis was utilized to describe the demographic and clinical characteristics of the sample and then univariate linear regression was used to find the effect of different predictors on CBCL Syndromic Scales while logistic regression was used to find the effect of different predictors on psychiatric diagnostic categories, and finally, univariate linear regression was used again to evaluate the predictor value of sexual abuse previously experienced by the parents for the parenting styles they practiced with their offspring. Significance of the obtained results was judged at the $5 \%$ level.

\section{Results}

As presented in Table 1, our sample of children had a mean age of $10.8 \pm 2.8$ years with a female representation of about $30 \%$ of the sample. Most of the children (85\%) were accompanied by their mothers and almost half $(50.7 \%)$ of the children had borderline intellectual abilities (IQ between 70 and 90). The most common diagnostic categories among the offspring were attention deficit hyperactivity disorder (ADHD) (54.1\%), elimination disorders (16.4\%), disruptive behavior disorders (14.5\%), and depression (13\%). Less common diagnoses included anxiety disorders (3.9\%), tic disorders or stuttering (2.4\%), OCD (1.9\%), bipolar disorder (1.4), and trauma-related disorders (1\%).

Table 2 shows the significant results of the univariate linear regression analysis of predictor variables for certain syndromic subscales of the CBCL as outcome variables. Unsurprisingly, our results highlight the significant effects of the family's socioeconomic status (SES) and child's IQ in predicting both child's competence and level of problematic behavior reported. Higher

\begin{tabular}{|c|c|}
\hline$(n=207)$ & \\
\hline \multicolumn{2}{|l|}{ Demographic variables } \\
\hline Age (mean \pm SD) & $10.8 \pm 2.8$ \\
\hline Female parents $(\mathrm{N}-\%)$ & $176(85 \%)$ \\
\hline Female children $(N-\%)$ & $64(30.9 \%)$ \\
\hline SES (mean \pm SD) & $47.7 \pm 12.1$ \\
\hline IQ median (min.-max.) & $84(71-116)$ \\
\hline \multicolumn{2}{|l|}{ Diagnostic category } \\
\hline Borderline intellectual functioning (BIF) ( $N-\%)$ & $105(50.7 \%)$ \\
\hline ADHD (N-\%) & $112(54.1 \%)$ \\
\hline Elimination disorders $(\mathrm{N}-\%)$ & $34(16.4 \%)$ \\
\hline Disruptive behavior disorders ( $N-\%)$ & $30(14.5 \%)$ \\
\hline Depression $(N-\%)$ & $27(13 \%)$ \\
\hline Anxiety disorders $(\mathrm{N}-\%)$ & $8(3.9 \%)$ \\
\hline Tic disorders $(\mathrm{N}-\%)$ & $5(2.4 \%)$ \\
\hline Stuttering $(N-\%)$ & $5(2.4 \%)$ \\
\hline Obsessive-compulsive spectrum (OCS) ( $N-\%)$ & $4(1.9 \%)$ \\
\hline Bipolar disorders $(\mathrm{N}-\%)$ & $3(1.4 \%)$ \\
\hline Trauma-related disorder ( $\mathrm{N}-\%)$ & $2(1 \%)$ \\
\hline
\end{tabular}




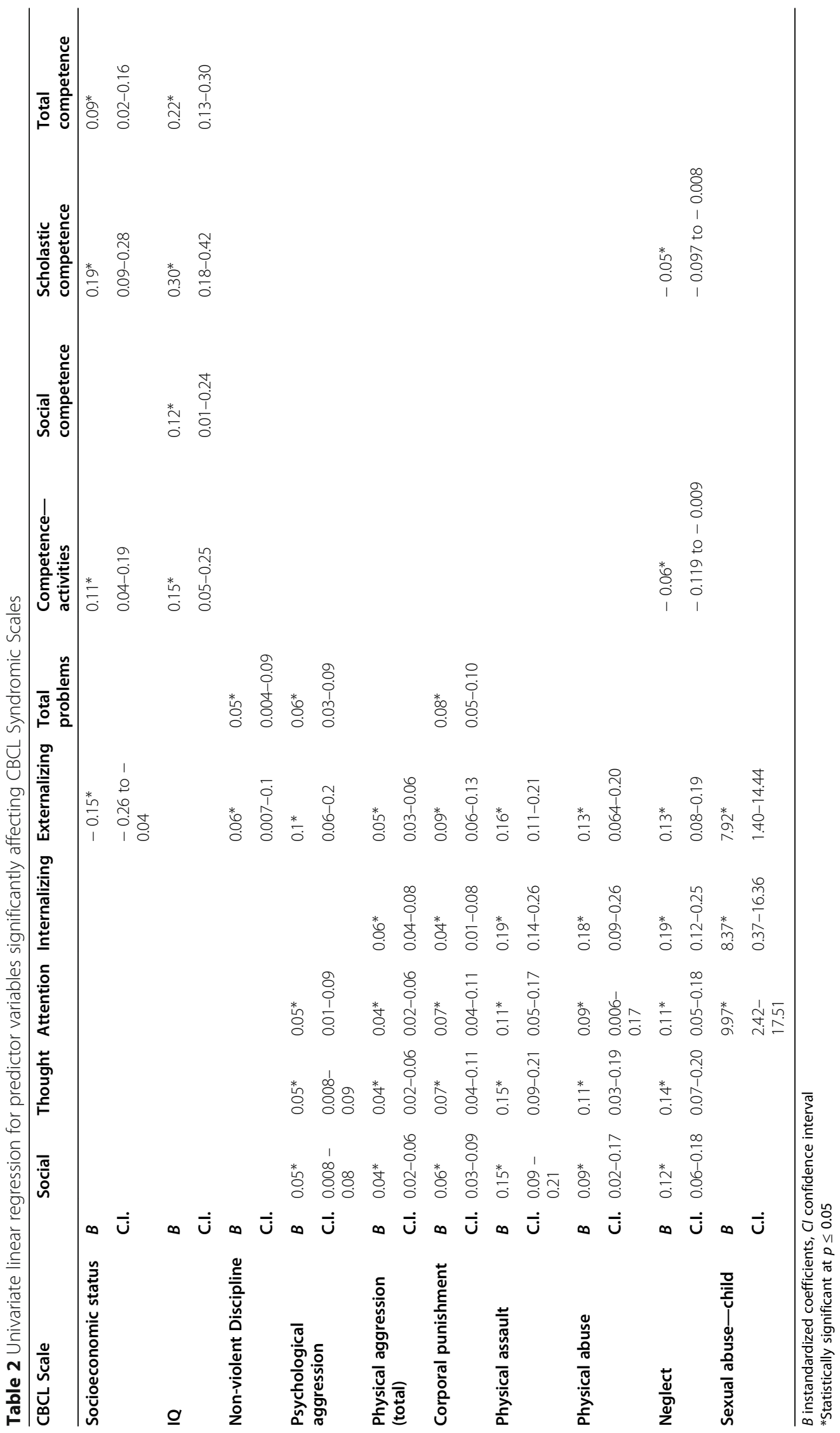


IQ and SES were both associated with better competence in scholastic achievement $(B=0.3$ for IQ and 0.19 for SES), leisure activities ( $B=0.15$ for IQ and 0.11 for SES), and total competence ( $B=0.22$ for IQ and 0.09 for SES), but only higher IQ was associated with better social performance $(B=0.12)$. Higher SES of the family did not predict better social competence but did predict fewer externalizing problems in the child $(B=-0.15)$.

Non-violent discipline practiced by the parents had the least significant contribution among other CTS-PC subscores in predicting externalizing $(B=0.06)$ and total behavioral problems $(B=0.05)$. The use of psychological aggression against the child, such as yelling, screaming, or threatening him/her), significantly predicted more social, thought, attention, externalizing, and total problems ( $B=0.05,0.05,0.05,0.1$, and 0.06$)$, respectively. The use of any type of physical punishment against the child significantly predicted more internalizing (Fig. 1) and externalizing (Fig. 2) problems $(B=0.06,0.05)$ in addition to more social, thought, and attention problems $(B=0.04$, 0.04, 0.04), respectively. Breaking up physical aggression into three levels of severity, namely corporal punishment, physical assault, and physical maltreatment, did not yield different results. In other words, physical punishment predicted the same outcomes regardless of the severity of the assault. The neglect of the child also predicted more social $(B=0.12)$, thought $(B=0.14)$, attention $(B=0.11)$, internalizing $(B=0.19)$, and externalizing problems $(B=0.13)$ in addition to less competence in activities $(B=-0.06)$ and scholastic activities $(B=-0.05)$ of the child. Positive history for sexual abuse experienced by the child had a much higher predictor effect on attention $(B=9.97)$, internalizing ( $B$ $=8.37)$, and externalizing problems $(B=7.92)$.

The predictor value of the various discipline styles on KSADS-PL diagnostic categories was then explored using logistic regression (Table 3, Fig. 3). Children with higher SES were more likely to receive the diagnosis of anxiety disorders $(\mathrm{OR}=1.19)$. Neglect during the last year predicted the diagnosis of disruptive behavior disorders $(\mathrm{OR}=1.2)$. On the other hand, the diagnosis of stuttering was predicted by lifetime occurrence of psychological aggression $(\mathrm{OR}=2.34)$. Further analysis of multiple levels of severity of physical aggression revealed that the predictor effect came mainly from moderate aggression (physical assault) and not from mild or severe cases. The diagnosis of tic disorders was more likely to be given to children subjected to lifetime exposure to neglect $(\mathrm{OR}=7.64)$.

Finally, univariate linear regression revealed that sexual abuse experienced by the parents when they were children predicted more physical aggression, especially moderate and severe forms, practiced by them on their own children $(\mathrm{OR}=48.65)$ (Table 4, Fig. 4). Parents subjected to sexual abuse were also more likely to neglect their own children as parents $(\mathrm{OR}=20.85)$.

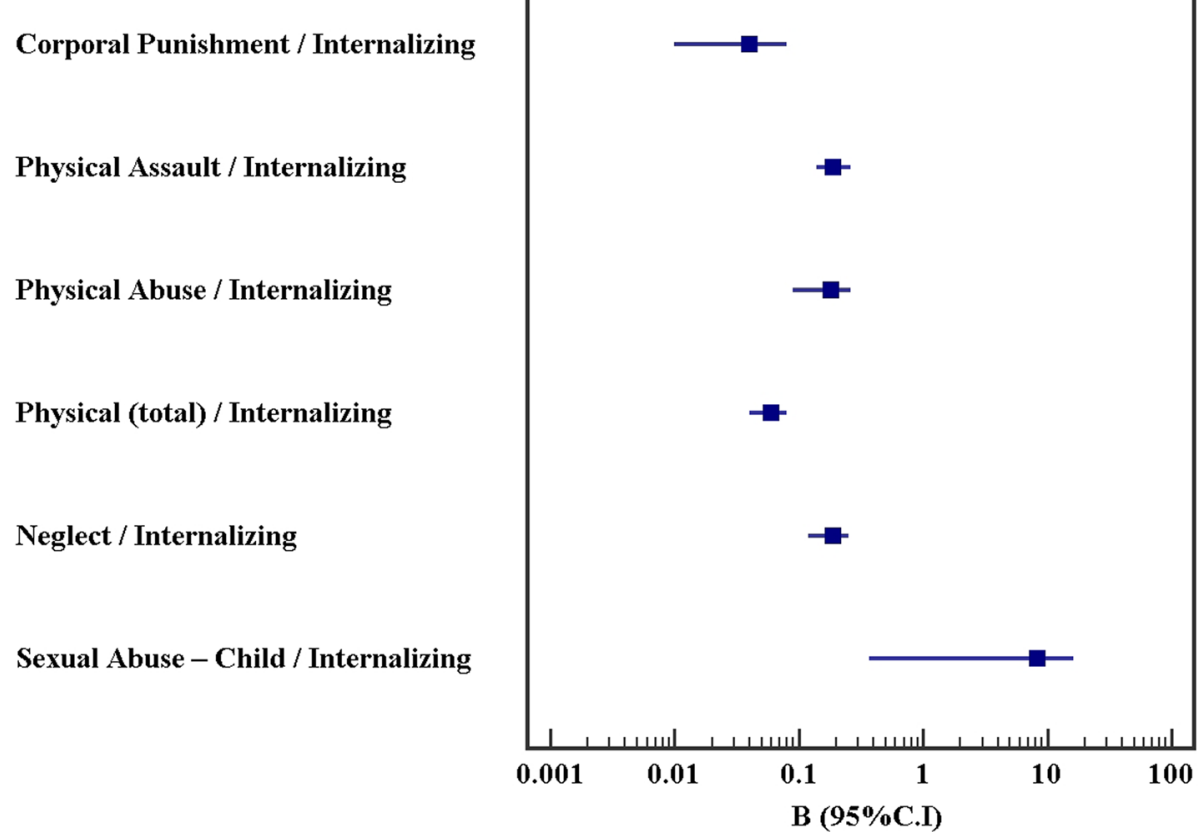

Fig. 1 Univariate linear regression for predictor variables significantly affecting $C B C L$ internalizing problems scale 


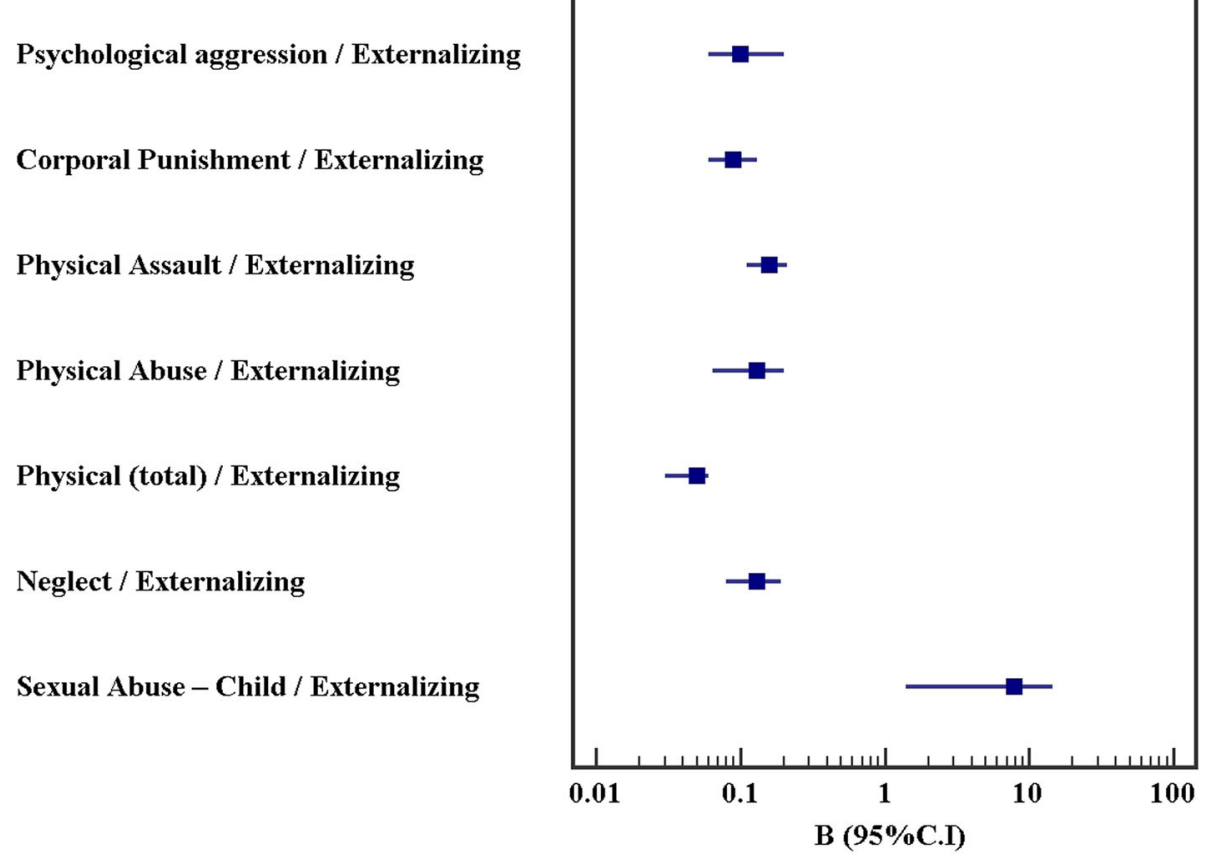

Fig. 2 Univariate linear regression for predictor variables significantly affecting $C B C L$ externalizing problems scale

\section{Discussion}

The current study reports robust positive association between all forms of psychological and physical violence against children and both internalizing and externalizing problems in offspring with no significant differences between milder forms of corporal punishment, classically accepted in our culture, and severer forms of violence and abuse. Neglect had a similar impact on the psychological well-being of the children with further detrimental effect on children's competence. Non-violent discipline had the least significant role in increasing externalizing problems in our sample and no significant effect on internalizing problems. Sexually abused children were more likely to have internalizing and externalizing problems while parents who were sexually abused as children had more tendency to practice violent discipline strategies on their own children.

The predictive value of IQ in the child's performance noticed in our results is a well-established and frequently reported result [37-40]. Our findings also supported the direct effect of the family's SES on externalizing problems. These results replicate what was previously

Table 3 Logistic regression for predictor variables significantly affecting psychiatric diagnostic categories

\begin{tabular}{|c|c|c|c|c|c|c|c|c|c|c|c|}
\hline Diagnosis & & ADHD & Disruptive & Elimination & TRD & OCS & Depression & BP & Anxiety & Tics & Stuttering \\
\hline \multirow[t]{3}{*}{ SES } & OR & & & & & & & & $1.19^{*}$ & & \\
\hline & C.I. & & & & & & & & $1.07-1.31$ & & \\
\hline & C.I. & & & & & & & & & & \\
\hline \multirow[t]{2}{*}{ Psychological aggression $-\mathrm{L}$} & OR & & & & & & & & & & $2.34^{*}$ \\
\hline & C.I. & & & & & & & & & & $1.09-5.03$ \\
\hline \multirow[t]{2}{*}{ Physical Assault-L } & OR & & & & & & & & & & $2.83^{*}$ \\
\hline & C.I. & & & & & & & & & & $1.04-7.67$ \\
\hline \multirow[t]{2}{*}{ Neglect } & OR & & $1.02^{*}$ & & & & & & & & \\
\hline & C.I. & & $1.01-1.04$ & & & & & & & & \\
\hline \multirow[t]{2}{*}{ Neglect-L } & OR & & & & & & & & & $7.46^{*}$ & \\
\hline & C.I. & & & & & & & & & $2.03-27.46$ & \\
\hline
\end{tabular}

$O R$ odds ratio, $\mathrm{Cl}$ confidence interval, $L$ lifetime occurrence but not in the past year

*Statistically significant at $p \leq 0.05$ 


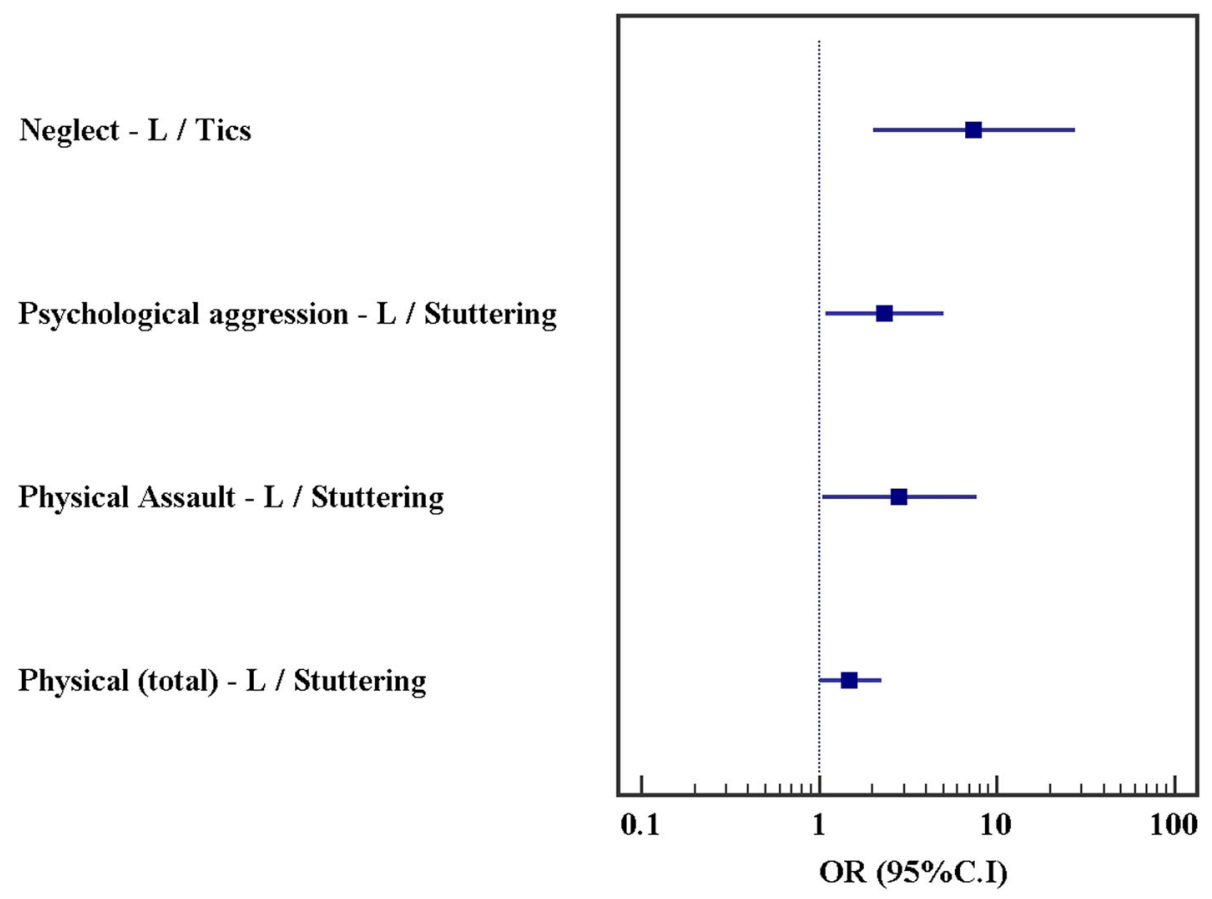

Fig. 3 Logistic regression for predictor variables significantly affecting psychiatric diagnostic categories

reported in several Western and Eastern cultures [4144]. Children coming from high SES families suffered more anxiety disorders, a finding that does not seem to replicate previously reported results which mostly link lower SES with higher rates of anxiety disorders [45-48]. This apparent contradiction might be attributed to the small number of children diagnosed with these diagnostic categories in our sample.

The relationship between certain parenting discipline styles and children's internalizing and externalizing behaviors is supported by several previous reports in several diverse cultures $[3,5-7,10,16,49-52]$. The current study reports a slightly significant relationship between the non-violent discipline of parents and the children's externalizing and total problems. This result goes along with the previously reported results [7] which noticed a low rate of psychiatric problems in children exposed to authoritative discipline. These findings may be explained by the multi-factorial pathogenesis of psychiatric disorders in which the relationship between any type of discipline and behavioral problems in children is mediated by genetic predisposition, in addition to other biological and social factors.

According to the UNICEF survey [16] in three major Egyptian cities, physical punishment was widely seen as a legitimate disciplinary method by children, parents, and teachers. The current study emphasizes the negative effects of corporal punishment on children's well-being confirming what was reported in other cultures [11, 5356]. The results of the current study contradict and might help to correct the common belief that mild physical violence is not psychologically harmful to children. The current study has also reported a significant association between parental psychological aggression and the

Table 4 Univariate linear regression for sexual abuse experienced by parents when they were children affecting their parenting behaviors with their own children

\begin{tabular}{lll}
\hline Outcome & \multicolumn{2}{l}{ Sexual abuse experienced by the parent when he/she was a child } \\
\cline { 2 - 3 } & $\boldsymbol{p}$ & $\boldsymbol{B}(\mathbf{9 5 \%} \mathrm{Cl})$ \\
\hline Physical aggression (total) & $0.019^{*}$ & $48.65(8.096-89.193)$ \\
Physical assault & $0.006^{*}$ & $17.69(5.158-30.211)$ \\
Physical abuse & $0.029^{*}$ & $11.26(1.190-21.321)$ \\
Neglect & $0.001^{*}$ & $20.85(8.836-32.866)$ \\
\hline
\end{tabular}

$B$ unstandardized coefficients, $\mathrm{Cl}$ confidence interval

*Statistically significant at $p \leq 0.05$ 


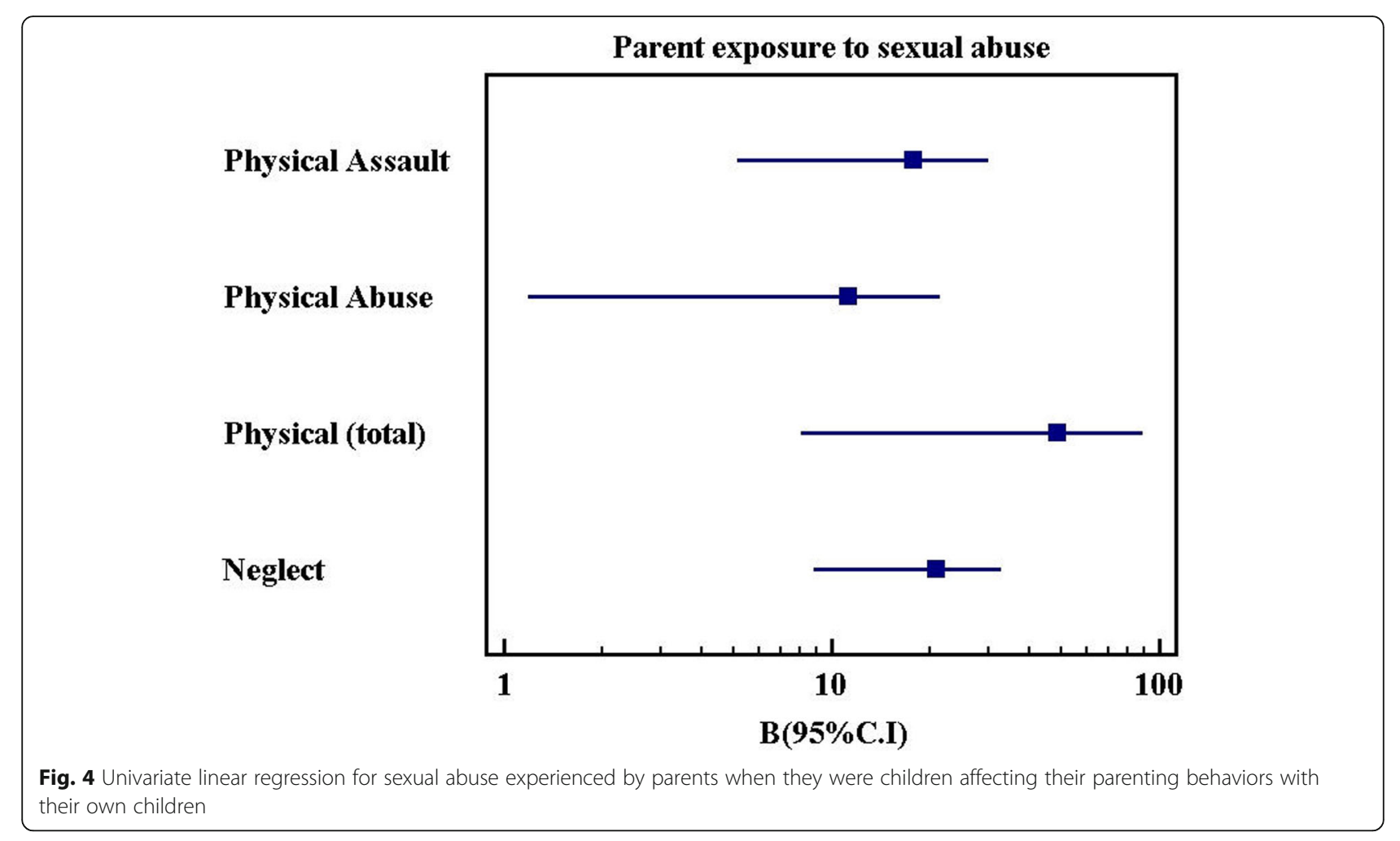

social, thought, attention, and externalizing problems in meticulous children. The same findings were previously reported by McKee and colleagues [57] who reported that harsh verbal parenting had a direct effect on both internalizing and externalizing behavior of youth. Other researchers [58] also documented that rejecting behavior of parents and inconsistent parenting were associated with aggression and anti-social behavior in children.

In the current study, child neglect was linked to social, thought, and attention problems as well as externalizing and internalizing disorders. These results replicate the results of several studies $[59,60]$ which reported a negative relationship between the degree of parental monitoring and the intensity of childhood socio-emotional and behavioral problems. However, Stevens and his colleagues [4] have reported a significant relationship between parental care and behavioral problems in adolescents only, and not in children. Our study confirmed the multiple previous findings that emphasize the powerful significant association between sexual abuse and externalizing problems and internalizing problems of children and adolescents [61-65].

We also report that children treated with neglect were more likely to be diagnosed with disruptive behavior disorders, a result that was previously reported in several other cultures [66-68]. Children who have previously received aggressive treatment, both psychologically and physically, were more likely to receive the diagnosis of stuttering. Again, several previous studies, including some studies carried out in the Arab countries [69-71], reported the direct correlation between violent, rejecting, and neglecting parenting behaviors and childhood stuttering. Previous lifetime neglect was also associated with the diagnosis of tics. This finding, not reported before in the literature, might reflect the psychopathological role of tics in drawing the attention of neglectful parents to the child. A previous study [72] reported a decreased rate of tics in maltreated children and attributed this result to the possible trial of the children to suppress their tics when risking punishment for them by their parents.

According to the World Health Organization [73], about $20 \%$ of women and $5-10 \%$ of men have been sexually abused as children. To our knowledge, this is the first study to explore the parenting experience of Arab-speaking parents who had been sexually abused when they were children. We report a much higher probability of these parents to use more violent discipline styles, such as psychological and physical aggression against their own children in addition to a much higher probability to be more neglectful toward these children. Some previous studies reported a significant relationship between child sexual abuse and lower parenting selfefficacy and more permissive parenting [74] while other studies suggested that parenting is not generally problematic in previously abused parents and even might be a healing experience for them $[75,76]$. These conflicting 
results call for further research to investigate the relationship between physical and sexual abuse of children and their parenting behavior.

Before we conclude, we would like to discuss the limitations of this study. First, the relatively small clinical sample might make it difficult to generalize the results of this study on Egyptian society. Larger community samples are needed to explore the patterns of parenting among the general population. Second, the collection of parent reports about their children without self-reports or teacher reports might subject the results to personal attitudes and bias from parents when judging their own behavior and the behavior of their children. Third, the current cross-sectional design of the study could not exclude the possible two-directional association between violent parenting and child psychopathology. Future prospective studies are crucially needed to confirm that the onset of harsh violent parenting predates the onset of child psychopathology. Fourth, the exclusion of parents with psychiatric disorders and children with intellectual disability, autism spectrum disorder, or psychotic disorders might be considered as a selection bias that led to a better than real world results. Fifth, different comorbidities between disorders were not calculated as the study was based only on the primary diagnosis of each case. The predominance of the male gender and the diagnosis of ADHD might make it difficult to extrapolate the result on larger community samples. Finally, the absence of a comparison control group might add to the limitations of the current study. A case-control study design needed to explore our hypothesis would be to have a control group with absolutely no use of parenting violent styles, which is very hard to recruit in our culture, and investigate the levels of psychopathology in their offspring.

\section{Conclusions}

This study has investigated different parenting disciplines in a sample of Egyptian children and concluded that all forms of child maltreatment, even mild forms, are associated with increased levels of internalizing and externalizing problems in offspring. These findings may be helpful in the future to diminish the risk of psychopathology in youth in Arab Egyptian society by suggesting specially designed parenting programs to educate parents on how to deal with their children's problems and to early identify children exposed to negative parenting. National policies and legalization should be implemented to decrease child maltreatment and protect children from all forms of physical and sexual abuse.

\section{Abbreviations}

ADHD: Attention deficit-hyperactivity disorder; CBCL 6-18: Child behavioral checklist questionnaire for ages 6-18; CTS-PC: Conflict tactics scale-parent child version; DBD: Disruptive behavior disorders; DSM-5: Diagnostic and
Statistical Manual of Mental Disorders-version 5; IBM: International Business Machines; ID: Intellectual disability; IQ: Intelligence quotient; K-SADSPL: Kiddie Schedule of affective disorders and schizophrenia-present and lifetime version; MINI-Kid: MINI International Neuropsychiatric Interview for Children and Adolescents for parents and with children and adolescents; OCD: Obsessive-compulsive disorder; OR: Odds ratio; SPSS: Statistical Package for the Social Sciences; UNICEF: United Nations International Children's Emergency Fund

\section{Acknowledgements}

Many thanks to Professor Dr, Ibrahim Kabbash, MD, Professor of Public Health \& Community Medicine, Faculty of Medicine, Tanta University, for helping us in calculating the sample size and power analysis. We would also like to express our deep appreciation to Ahmed El-Zawawy, clinical psychologist, who did the psychometric evaluation for the recruited adolescents, and to Maha Salah and Marwa Salah, the social workers who conducted the socio-economic evaluation for participating families. Thanks are due also to the whole nursing and technician teams in the Child and Adolescent Psychiatry unit at Tanta Psychiatry and Neurology. All thanks and gratitude are also due to our families and parents.

\section{Authors' contributions}

MS played a major role in conceptualizing the aim and hypothesis of this work, confirming the diagnoses of all recruited subjects, analyzing the data, and writing the manuscript. RA had a substantial contribution in recruiting and interviewing families, reviewing the literature, and shared in writing the manuscript. Both authors approved the submitted version (and any substantially modified version that involves the author's contribution to the study).

Funding

This study was self-funded. No funds were received.

\section{Availability of data and materials}

The dataset created and analyzed during the current study will be available from the corresponding author on reasonable request.

Ethics approval and consent to participate

A written consent from parents and a verbal assent from the child were obtained prior to participation in the study. The study was approved by the Ethical Research Committee of the Faculty of Medicine, Tanta University, under the code 31892/11/17.

Consent for publication

Not applicable.

\section{Competing interests}

All authors report no biomedical financial interests or potential conflicts of interest.

Received: 6 August 2020 Accepted: 13 October 2020

Published online: 28 October 2020

\section{References}

1. Lorence B et al (2019) The role of parenting styles on behavior problem profiles of adolescents. Int J Environ Res Public Health 16(15):2767

2. Daly M, Bray R, Bruckauf Z, Byrne J, Margaria A, Pécnik N, Samms-Vaughan M (2015) Family and parenting support: policy and provision in a global context; innocenti insight. UNICEF Office of Research, Florence

3. Van As NMC, Janssens JMAM (2002) Relationships between child behaviorproblems and family functioning: a literature review. Int J Child Fam Welf 1-2:40-51

4. Stevens GWJM, Vollebergh WAM, Pels TVM, Crijnen AAM (2007) Parenting and internalizing and externalizing problems in Moroccan immigrant youth in the Netherlands. J Youth Adolesc 36(5):685-695

5. Sangawi H, Adams J, Reissland N (2018) The impact of parenting styles on children developmental outcome: the role of academic self-concept as a mediator. Int J Psychol 53(5):379-387

6. M ASATMARM (2011) Relationship between parenting style and children's behavior problems. Asian Soc Sci 7(12):195 
7. Pinquart M (2017) Associations of parenting dimensions and styles with externalizing problems of children and adolescents: an updated metaanalysis. Dev Psychol 53(5):873-932

8. Baumrind D (1967) Child care practices anteceding three patterns of preschool behavior. Genet Psychol Monogr 75(1):43-88

9. Gamez-Guadix M et al (2010) Corporal punishment and long-term behavior problems: the moderating role of positive parenting and psychological aggression. Psicothema 22(4):529-536

10. Pace GT, Lee SJ, Grogan-Kaylor A (2019) Spanking and young children's socioemotional development in low- and middle-income countries. Child Abuse Negl 88:84-95

11. Gershoff ET, Grogan-Kaylor A (2016) Spanking and child outcomes: old controversies and new meta-analyses. J Fam Psychol 30(4):453-469

12. Huntsinger CS, Jose PE (2009) Relations among parental acceptance and control and children's social adjustment in Chinese American and European American families. J Fam Psychol 23(3):321-330

13. Lansford JE et al (2004) Ethnic differences in the link between physical discipline and later adolescent externalizing behaviors. J Child Psychol Psychiatry 45(4):801-812

14. Chao RK (2001) Extending research on the consequences of parenting style for Chinese Americans and European Americans. Child Dev 72(6):1832-1843

15. Alsheikh, N., Parameswaran, G., \& Elhoweris, H. 2010, Parenting style, selfesteem and student performance in the United Arab Emirates. Current Issues in Education, 13(1).

16. UNICEF, N.C.f.C.a.M.N.a 2015., Violence against children in Egypt. A quantitative survey and qualitative study in Cairo, Alexandria and Assiut, N.a. U. Egypt, Editor:: Cairo

17. M A (2003) Children disciplining within the family context: reality and attitudes. Arabic childhood 16(4):9-38

18. M H (1974) Studies in youngs' socialization styles. Culture House for Printing and Publication, Cairo

19. H A-M (1981) Child education in Saudi Arabia attitudes. Sciences' House for Printing and Publication, Riyadh

20. Discala C et al (1998) Injuries to children with attention deficit hyperactivity disorder. Pediatrics 102(6):1415-1421

21. Soltan M (1986) Aletegahat alwaldyia wa elaqateha belsolok alegtemaai lelatfal, in Developmental Psychology. Cairo, Ain Shams

22. Muhriz N, Ahmad A, Al-Atum A (2005) Asaalieb Almaamala alwalidaya wa elaqatuha biltawafuq Iteefl alejtimae wa shakhsi fe ryad atffal. Parenting styles and their relationship with child's social and personal adjustment in Kindergarten. Mujala jamia Dimashq 12:285-324

23. Maarof, A. 1987, Asaleeb Alomhat fi altatbee alegtemaai leltefl fi alosra algazaeryia: Dar Alforqan. 324

24. Seleem MA et al (2019) Demographic and clinical characteristics of children seeking psychiatric services in the Nile Delta region: an observational retrospective study. Int J Ment Health Syst 13:66

25. Melika L (1998) The Stanford Binet Intelligence Scale, in Arabic Examiner's Handbook. Cairo, Dar El Maref Publishing

26. Thorndike RL, Hagen EP, Sattler JM (1986) Stanford-Binet Intelligence Scale, 4th edn. Riverside, Chicago

27. Fahmy SI, El Sherbini AF (1983) Determining simple parameters for socia classifications for health research. Bulletin of the High Institute of Public Health 13:95-108

28. El-Gilany A, El-Wehady A, El-Wasify M (2012) Updating and validation of the socioeconomic status scale for health research in Egypt. East Mediterr Health J 18(9):962-968

29. Kaufman, J.B., Boris, Brent, David, Rao, Uma, Ryan, Neal Diagnostic Interview: Kiddie-Sads-Present and Lifetime Version (PDF). 1996 2016-09-08.]

30. Ibrahim MBZ, H A. 2002 Comparison of Mini International Neuropsychiatriclnterview for children (MINI-KID) with the schedules for affective disorders and schizophrenia for schoolaged children, present and lifetime version (KSADS-PL) in an Egyptian sample presenting with childhood disorders, in Psychiatry. Ain Shams University: Cairo.

31. Straus MA, Hamby SL, Finkelhor D, Moore DW, Runyan D (1998) Identification of child maltreatment with the parent-child conflict tactics scales: development and psychometric data for a national sample of American parents. Child Abuse Negl 22(4):249-270

32. Straus MAH, Sherry L (1997) Measuring Physical and Psychological Maltreatment of Children with the Conflict Tactics Scales. In: Kantor GK, Jasinski JL (eds) Out of the darkness: contemporary research perspectives on family violence. Sage, Thousand Oaks, CA, pp 119-135
33. Straus MA (1979) Measuring intra family conflict and violence: The Conflict Tactics (CT) Scales. J Marriage Fam 41(1):75-88

34. Seleem M, A, El-Seht R, Saada SA (2016) Parenting discipline styles and child psychopathology in a sample of Egyptian children with accidental ocular trauma: a case-control study. Middle East Current Psychiatry 23:188-193

35. Achenbach TM, Rescorla LA (2001) Manual for the ASEBA School-Age Forms \& Profiles. Burlington: University of Vermont, Research Center for Children, Youth, \& Families

36. IBM Corp. Released 2011. IBM SPSS Statistics for Windows, Version 20.0. Armonk: IBM Corp

37. Akiyama $\mathrm{K}$ et al (2016) Predictive value of premorbid IQ, negative symptoms, and age for cognitive and social functions in Japanese patients with schizophrenia: a study using the Japanese version of the Brief Assessment of Cognition in Schizophrenia. Psychiatry Res 246:663-671

38. Middel RG et al (2018) The predictive value of amplitude-integrated electroencephalography in preterm infants for IQ and other neuropsychological outcomes at early school age. Neonatology 113(4):287295

39. Buelow JM et al (2003) Behavior and mental health problems in children with epilepsy and low IQ. Dev Med Child Neurol 45(10):683-692

40. Sammallahti $S$ et al (2017) Growth after late-preterm birth and adult cognitive, academic, and mental health outcomes. Pediatr Res 81(5):767-774

41. Anton MT, Jones DJ, Youngstrom EA (2015) Socioeconomic status, parenting, and externalizing problems in African American single-mother homes: a person-oriented approach. J Fam Psychol 29(3):405-415

42. Besser A, Dohnert M, Stadelmann S (2019) Various socioeconomic factors as predictors of internalizing and externalizing disorders in children and adolescents. Z Kinder Jugendpsychiatr Psychother 47(4):345-358

43. Scaramella LV et al (2008) Consequences of socioeconomic disadvantage across three generations: parenting behavior and child externalizing problems. J Fam Psychol 22(5):725-733

44. Hosokawa R, Katsura T (2018) Effect of socioeconomic status on behavioral problems from preschool to early elementary school - a Japanese longitudinal study. PLoS One 13(5):e0197961

45. Koenen KC et al (2009) Childhood IQ and adult mental disorders: a test of the cognitive reserve hypothesis. Am J Psychiatry 166(1):50-57

46. Zhu Y et al (2019) Socioeconomic status disparities affect children's anxiety and stress-sensitive cortisol awakening response through parental anxiety. Psychoneuroendocrinology 103:96-103

47. Gilman SE (2002) Childhood socioeconomic status, life course pathways and adult mental health. Int J Epidemiol 31(2):403-404

48. Gilman SE et al (2002) Socioeconomic status in childhood and the lifetime risk of major depression. Int J Epidemiol 31(2):359-367

49. Reid JB, Patterson GR, Snyder J (2002) Antisocial behavior in children and adolescents: a developmental analysis and model for intervention. American Psychological Association, Washington, DC

50. Ho C, Bluestein DN, Jenkins JM (2008) Cultural differences in the relationship between parenting and children's behavior. Dev Psychol 44(2): 507-522

51. Sprang G, Clark JJ, Bass S (2005) Factors that contribute to child maltreatment severity: a multi-method and multidimensional investigation. Child Abuse Negl 29(4):335-350

52. Goraya, F., \& Shamama-tus-Sabah, S. 2013. Parenting, children's behavioral problems, and the social information processing among children. Pak J Psychol Res, 28(1):107-111.

53. Larzelere RE (2000) Child outcomes of nonabusive and customary physical punishment by parents: an updated literature review. Clin Child Fam Psychol Rev 3(4):199-221

54. Taylor CA et al (2010) Mothers' spanking of 3-year-old children and subsequent risk of children's aggressive behavior. Pediatrics 125(5):e1057e1065

55. Mackenbach JD et al (2014) Exploring the relation of harsh parental discipline with child emotional and behavioral problems by using multiple informants. The generation R study. PLoS One 9(8):e104793

56. Fletcher AC, Walls JK, Cook EC, Madison KJ, Bridges TH (2008) Parenting style as a moderator of associations between maternal disciplinary strategies and child well-being. J Fam Issues 29(12):1724-1744

57. McKee L, Roland E, Coffelt N, Olson AL, Forehand R, Massari C, Zens MS (2007) Harsh discipline and child problem behaviors: the roles of positive parenting and gender. J Fam Violence 22(4):187-196 
58. Gao Y, Zhang W, Fung AL (2015) The associations between parenting styles and proactive and reactive aggression in Hong Kong children and adolescents. Int J Psychol 50(6):463-471

59. Michiels D, Grietens H, Onghena P, Kuppens S (2008) Parent- child interactions and relational aggression in peer relationships. Developmental Review 28:522-540

60. Yang HJ et al (2014) Culture, parenting, and child behavioral problems: a comparative study of cross-cultural immigrant families and native-born families in Taiwan. Transcult Psychiatry 51(4):526-544

61. Singh MM, Parsekar SS, Nair SN (2014) An epidemiological overview of child sexual abuse. J Family Med Prim Care 3(4):430-435

62. Krug EG, Dahlberg L, Mercy JA, Zwi AB, Lozano R (2002) World report on violence and health. World Health Organization, Geneva

63. Miller KL, D M, Miller SM (2007) A counselor's guide to child sexual abuse: prevention, reporting and treatment strategies

64. Putnam FW (2003) Ten-year research update review: child sexual abuse. J Am Acad Child Adolesc Psychiatry 42(3):269-278

65. Collin-Vezina D, Daigneault I, Hebert M (2013) Lessons learned from child sexual abuse research: prevalence, outcomes, and preventive strategies. Child Adolesc Psychiatry Ment Health 7(1):22

66. Grasso DJ et al (2016) Harsh parenting as a potential mediator of the association between intimate partner violence and child disruptive behavior in families with young children. J Interpers Violence 31(11):2102-2126

67. Liu L, Wang M (2015) Parenting stress and harsh discipline in China: the moderating roles of marital satisfaction and parent gender. Child Abuse Negl 43:73-82

68. Liu L, Wang M (2015) Parenting stress and children's problem behavior in China: the mediating role of parental psychological aggression. J Fam Psychol 29(1):20-28

69. Abu-Zeid HYR, A-Z bA (2012) The effect of domestic violence on speech and pronunciation disorders concerning children at the basic stage of education at Ajloun Governorate in Jordan. IOSR Journal of Humanities and Social Science (IOSRJHSS) 1(3):50-59

70. Eshghizadeh M, M M, Beydokhti TB, Banafshe E, Najafi S, kiyani A (2014) Comparison of parenting style in mothers of children with and without stuttering: a case control study. J Res Health 4(1):623-629

71. A GJ (1998) A study of some environmental and psychological variables associated with the phenomenon of stuttering in speech in children, in Graduate Institute of Childhood. Ain Shams University, Cairo

72. Dinkler $L$ et al (2017) Maltreatment-associated neurodevelopmental disorders: a co-twin control analysis. J Child Psychol Psychiatry 58(6):691701

73. Organization, W.H, Preventing child maltreatment: a guide to taking action and generating evidence. 2006.

74. Jaffe AE, Cranston CC, Shadlow JO (2012) Parenting in females exposed to intimate partner violence and childhood sexual abuse. J Child Sex Abus 21(6):684-700

75. Wark J, Vis JA (2018) Effects of child sexual abuse on the parenting of male survivors. Trauma Violence Abuse 19(5):499-511

76. Fujiwara T, Okuyama M, Izumi M (2012) The impact of childhood abuse history, domestic violence and mental health symptoms on parenting behaviour among mothers in Japan. Child Care Health Dev 38(4):530-537

\section{Publisher's Note}

Springer Nature remains neutral with regard to jurisdictional claims in published maps and institutional affiliations.

\section{Submit your manuscript to a SpringerOpen ${ }^{\circ}$ journal and benefit from:}

- Convenient online submission

- Rigorous peer review

- Open access: articles freely available online

- High visibility within the field

- Retaining the copyright to your article

Submit your next manuscript at $\boldsymbol{\nabla}$ springeropen.com 